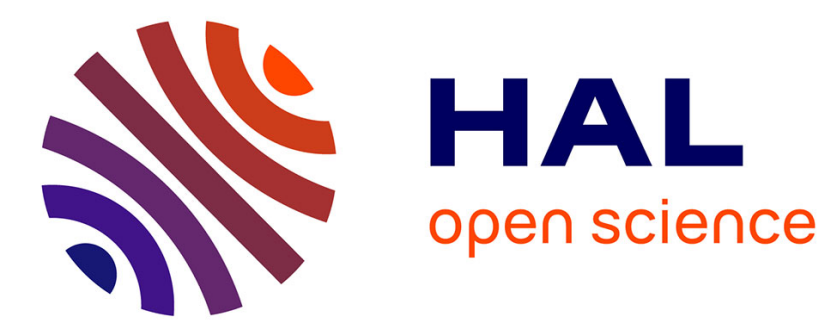

\title{
Anticiper et évaluer l'utilité dans la conception ergonomique des technologies émergentes: une revue
}

Emilie Loup-Escande, Jean-Marie Burkhardt, Simon Richir

\section{To cite this version:}

Emilie Loup-Escande, Jean-Marie Burkhardt, Simon Richir. Anticiper et évaluer l'utilité dans la conception ergonomique des technologies émergentes: une revue. Le travail humain, 2013, 76 (1), pp.27-55. 10.3917/th.761.0027 . hal-02451495

\section{HAL Id: hal-02451495 \\ https://hal.science/hal-02451495}

Submitted on 23 Jan 2020

HAL is a multi-disciplinary open access archive for the deposit and dissemination of scientific research documents, whether they are published or not. The documents may come from teaching and research institutions in France or abroad, or from public or private research centers.
L'archive ouverte pluridisciplinaire HAL, est destinée au dépôt et à la diffusion de documents scientifiques de niveau recherche, publiés ou non, émanant des établissements d'enseignement et de recherche français ou étrangers, des laboratoires publics ou privés. 


\title{
ANTICIPER ET ÉVALUER L'UTILITÉ DANS LA CONCEPTION ERGONOMIQUE DES TECHNOLOGIES ÉMERGENTES : UNE REVUE
}

\author{
par Émilie Loup-EsCANDE ${ }^{\star}$, JeAN-MARIE BURKHARDT ${ }^{\star \star}$, SimON RICHIR ${ }^{\star \star \star}$
}

SUMMARY

ANTICIPATING AND EVALUATING THE USEFULNESS OF EMERGING TECHNOLOGIES IN ERGONOMIC DESIGN: A REVIEW OF USEFULNESS IN DESIGN

The overall aim of this paper is to outline a review of the concept of usefulness. In particular, it looks at the facets of usefulness and the methods and tools that could help to develop a useful system from the perspective of ergonomics. Firstly, we present the research context and explain our motivations for focusing on the usefulness of innovative systems. One reason is that there is no theoretical or methodological framework that explicitly addresses usefulness as a guide to design and define goals, in contrast with the concept of usability. The first section aims to clarify the concept of usefulness. We have highlighted two dimensions of usefulness: purpose-usefulness and value-usefulness. Purpose-usefulness relates to the functional and non-functional features of the artefact. Value-usefulness relates to the improvements or significant benefits that the artefact can bring to users. We then clarify the relationship between usefulness and requirements. The requirement is the original inspiration, the argument and the justification associated with the usefulness of an artefact during the design process. The second section presents usefulness from the point of view of ergonomics. First, we show the extent to which usefulness is a built and progressive feature of systems by revealing two worlds of usefulness: the prospective world and the retrospective world. The prospective world gathers all features, requirements and thinkable (although not necessarily desirable) solutions; the retrospective world puts together all the relevant experiences in terms of usefulness to inform designers of the artefact. We then specify the relationship between usefulness and other criteria of ergonomics, such as usability or acceptability. The third section provides an overview of methods and their contributions to the different facets of usefulness. We detail the contribution of methods to the production of a hypothesis and the development of prospective worlds, before moving on to prioritization, selection and the decision-making process. Finally, we examine the evaluation of usefulness. In this part, we discuss the links between the more or less conscious nature of the requirements, the two

ä Angers Univ., Arts et Metiers ParisTech, LAMPA, 2 bd du Ronceray, 49000 ANGERS, France - email : emilie.loup-escande@ensam.eu

ää IFSTTAR - Laboratoire de Psychologie de la Conduite, 2â allée des Marronniers, Satory 78000 VERSAILLES, France - email : jean-marie.burkhardt@ifsttar.fr

äää Arts et Metiers ParisTech, LAMPA, 2 bd du Ronceray, 49000 ANGERS, France email : simon.richir@ensam.eu 
dimensions and the two worlds of usefulness. Our conclusion includes the most relevant elements for the clarification of what is underlying to the concept of usefulness in the context of the design of emerging technologies.

Keywords: Ergonomics, Usefulness, Requirements, Design, Evaluation, Emerging technologies.

\section{INTRODUCTION}

En ergonomie informatique, plusieurs critères généraux coexistent pour guider la conception et l'évaluation d'outils et d'applications mieux adaptés. Parmi ces critères, celui de l'utilisabilité est probablement le plus fréquemment représenté aujourd'hui, tant dans les études et les principes publiés qu'en ce qui concerne la normalisation et les méthodes de conception préconisées. À tel point qu'il n'est pas rare que l'ergonomie soit assimilée à cette seule dimension de l'utilisabilité (Burkhardt ï Sperandio, 2004). Pour certains auteurs, toutefois, le critère d'utilité est considéré comme premier. L'idée est en effet que même parfaitement utilisable, un objet inutile peut difficilement être qualifié d'ergonomique (Blandford, Green, Furniss ï Makri, 2007 ; Burkhardt i Sperandio, 2004 ; Nielsen, 1993). C'est d'ailleurs souvent l'observation d'inadéquations (en anglais "misfit"; Blandford et al., 2007) entre ce dont l'utilisateur a besoin et ce que fournit effectivement le "système " qui souligne " en creux » l'importance du caractère d'utilité réelle pour les utilisateurs finaux. La question qui découle concerne la façon dont cette dimension de l'utilité est abordée, conceptualisée et outillée en ergonomie, mais aussi au sein des autres disciplines impliquées dans la conception (design, ingénierie, marketing, économie, gestion etc.). Cette question émerge de façon récurrente dans la littérature (Grudin, 1992 ; Blandford et al., 2007 ; Lindgaard ï Parush, 2008) et en particulier dans plusieurs articles récents avec une focalisation sur une technologie spécifique comme l'informatique mobile (Wealands, 2006) ou sur une classe d'applications de la technologie comme l'utilisation du Web pour l'apprentissage (Silius ï Tervakari, 2003 ; Tricot et al., 2003).

Développer des recherches sur l'utilité des systèmes innovants nous semble répondre à quatre arguments principaux en termes d'intérêt tant pratique que théorique. Le premier est le risque de focaliser la conception et l'évaluation sur l'utilisabilité et de négliger l'utilité (Keil, Beranek ï Konsynski, 199â). En effet, même très utilisable, un système a de fortes chances de ne pas être accepté s'il n'est pas utile. La télévision interactive est un bon exemple de projet ayant souffert d'un manque d'utilité en dépit d'un effort important dédié à l'utilisabilité. Le second argument est relatif à l'idée que l'effort mis en œuvre pour améliorer l'utilité d'une application fournit un retour à long terme plus important que celui mis en œuvre pour perfectionner l'utilisabilité globale d'un système (Scholtz, Morse ï Steves, 2006). Le troisième argument est suggéré par un paradoxe (Anastassova, 2006) concernant la contribution de l'ergonomie à la conception des technologies émergentes. D'une part, il y a " un besoin fort de principes, de préconisations et de recommandations ergonomiques, à cause du caractère émergent de la technologie, et d'autre part, on a peu de réponses ergonomiques concrètes 
à fournir aux concepteurs, puisque le savoir de l'ergonomie s'appuie en grande partie sur l'analyse de défauts (...) observés antérieurement ou par ailleurs " (Anastassova, 2006, pp. 1â6, 1â7). Ces défauts peuvent être certes des défauts technologiques (Anastassova, 2006), mais également des défauts liés aux fonctionnalités qui sont encore imparfaitement cernées du fait du manque d'usage et de précédents (Burkhardt, 2006). Enfin, le quatrième argument est qu'il n'y a pas aujourd'hui - pour le praticien comme pour le chercheur en ergonomie - de cadre théorique ou méthodologique traitant explicitement de l'utilité pour guider la conception et définir les objectifs à atteindre, comparativement à la précision et la plus grande diffusion des cadres, des méthodes et des objectifs associés à l'utilisabilité. Cette difficulté provient aussi du fait que davantage d'études empiriques ont produit des préconisations en termes d'utilisabilité, alors qu'elles sont plus rares en ce qui concerne les contributions des méthodes à la dimension d'utilité.

L'objectif de cet article est par conséquent d'éclairer ce qui est sousjacent à la notion d'utilité dans le contexte de la conception et l'évaluation ergonomique, notamment dans le contexte des technologies émergentes. Il s'agit de tenter d'esquisser une synthèse sur la notion d'utilité, ses facettes et sur les façons dont les méthodes et les outils contribuent à concevoir un système "utile " selon la perspective de l'ergonomie. Loin de proposer un panorama définitif, il s'agit de poser les bases d'un questionnement réflexif fondé à la problématique d'une contribution de l'ergonomie à une conception centrée sur l'utilité.

La suite de l'article est organisée comme suit. Dans la première section, nous tentons de préciser la notion d'utilité au travers de deux dimensions, l'utilité-destination et l'utilité-valeur, ainsi qu'en clarifiant la relation qu'il existe entre l'utilité et les besoins. Dans la seconde section, nous expliquons en quoi l'utilité est une propriété construite et évolutive des systèmes; nous introduisons notamment les notions d'univers prospectif et d'univers rétrospectif, puis nous développons les liens qui existent entre l'utilité et d'autres critères de l'ergonomie. La troisième section propose une synthèse des méthodes et de leurs contributions aux différentes facettes de l'utilité. Nous détaillons la contribution des méthodes à la production d'hypothèses et à l'élaboration d'univers prospectifs, puis à la hiérarchisation, la sélection et la prise de décisions, et enfin à l'évaluation de l'utilité. Nous discutons également des liens entre le caractère plus ou moins conscient des besoins, les deux dimensions (utilité-destination et utilité-valeur) et les deux univers de l'utilité (prospectif et rétrospectif). En conclusion, nous reprenons les éléments les plus pertinents à la clarification de ce qui est sous-jacent à la notion d'utilité dans le contexte de la conception de technologies émergentes.

\section{VERS UNE CLARIFICATION DE LA NOTION D'UTILITÉ EN CONCEPTION}

Le terme "utilité ", comme ceux de besoins et de fonctions désirées, est couramment mobilisé sans qu'il existe une articulation claire entre les notions qu'ils recouvrent ni des acceptions communément acceptées. 
En lien avec la conception, le terme d'utilité est plutôt utilisé en ergonomie (Burkhardt ï Sperandio, 2004 ; Nielsen, 1993 ; Tricot et al., 2003) et en économie des nouvelles technologies (Turk, 2003). Le terme admet deux traductions possibles dans les publications en langue anglaise : " utility » versus " usefulness ». Le terme " utility ", surtout utilisé en sciences économiques (Neumann ï Morgenstern, 1947), est peu fréquent dans la littérature en conception. La traduction par « usefulness " est plus communément employée en conception, et en particulier dans la littérature sur les Interfaces Humain-Machine. D'autres termes aux significations proches ou partiellement connexes à l'utilité sont parfois aussi mobilisés, par exemple celui de pertinence (Tricot, 2001), ou encore d'adaptabilité ${ }^{1}$ et de compatibilité ${ }^{2}$ (Scapin $\ddot{i}$ Bastien, 1997). En ergonomie, ils se réfèrent généralement à l'idée que l'utilité est une propriété se situant à l'interface entre le système, le sujet et la situation, correspondant à une réponse à un besoin ou à une attente de l'utilisateur.

En Ingénierie, comme en marketing, on retrouve également les termes de " besoins ", " attentes " ou encore " exigence ». Utilisés dans des disciplines différentes, les besoins et les attentes ne renvoient pas systématiquement au même contenu. Les propriétés de l'artefact en termes de réponses aux besoins sont alors plutôt traduites par les termes de "fonctions " (Richir, 2003) ou encore de "spécifications techniques des besoins" (Nicolas, 2000). En marketing, c'est surtout l'utilisation du terme "attente" qui est récurrente. Olivier (1980) définit les attentes comme les prévisions des usages futurs ("predictions of future performance "). Spreng, Mackenzie et Olshavsky (1996) définissent les attentes comme des désirs ou des idéaux (" desires " ou "ideals ") conscients chez les consommateurs. Sheth (1973) définit les attentes comme le potentiel perçu par les fournisseurs des marques pour satisfaire des objectifs explicites et implicites dans toute décision d'achat particulière.

À cette hétérogénéité des significations et de la terminologie s'ajoute parfois aussi l'illusion de partager la même définition entre les acteurs et les disciplines de la conception. Il importe donc de commencer par clarifier la notion d'utilité dans le contexte de la conception.

\section{I.1. UNE DISTINCTION ENTRE L'UTILITÉ-DESTINATION (DE L'ARTEFACT) ET L'UTILITÉ-VALEUR (POUR L'UTILISATEUR)}

Clarifier cette notion d'utilité nous a amenés à considérer dans un premier temps la définition même du terme. Le Nouveau Petit Robert (2007) énonce en effet deux sens à la notion d'utilité. Le premier désigne "le caractère de ce qui est utile ", c'est-à-dire le caractère de "ce dont l'usage,

1. C'est-à-dire défini comme "la capacité de l'outil à réagir selon le contexte, et selon les besoins et préférences des utilisateurs ".

2. C'est-à-dire en ce sens de «l'accord pouvant exister entre les caractéristiques des utilisateurs (mémoire, perceptions, habitudes, compétences, âge, attentes, etc.) et des tâches, d'une part, et l'organisation des sorties, des entrées et du dialogue d'une application donnée, d'autre part ". 
l'emploi est ou peut être avantageux pour quelqu'un", ce qui "satisfait un besoin ». Dans ce premier sens, l'utilité est synonyme de "bon, profitable, salutaire, indispensable, nécessaire ". Le terme d'utilité désigne deuxièmement "ce en quoi un objet est utile ", c'est-à-dire la fonction qu'il remplit, le service qu'il fournit. Cette définition littéraire met ainsi en exergue deux facettes qui, bien que liées, peuvent être distinguées du point de vue de l'analyse : l'utilité comme la "plus-value " ou la "valeur" associée à l'emploi de cet objet pour celui qui l'utilise effectivement, et l'utilité comprise comme la "destination " associée à l'objet considéré.

En ergonomie, l'usage qui est fait du terme "utilité » oscille entre ces deux acceptions d'utilité-valeur et d'utilité-destination. Ainsi pour Burkhardt et Sperandio (2004), l'utilité (au sens de la valeur ou de la plus-value) se définit comme un avantage ou un agrément significatif de l'artefact pour l'utilisateur dans des activités médiatisées par un système informatique, cet avantage étant toujours relatif : relatif aux objectifs de l'utilisateur, relatif aux outils existants ou habituellement utilisés, relatif à l'environnement d'utilisation, aux dépendances avec les autres activités. En effet, pour l'ergonomie s'intéressant à l'amélioration des conditions de travail au sens large (en particulier - mais pas seulement - au travers d'outils plus "adaptés 》), le fait que l'artefact assiste effectivement l'utilisateur dans l'atteinte de ses objectifs ne constitue pas en soi un critère suffisant (en fait, c'est même un critère minimal pour tout outil ayant l'ambition d'une «bonne " ergonomie). Il est d'autre part nécessaire que les conditions dans lesquelles l'artefact permet d'atteindre les objectifs poursuivis constituent une amélioration réelle par rapport à l'existant. Les critères candidats à l'évaluation d'une telle amélioration sont nombreux, éventuellement en conflit les uns avec les autres, telles l'efficacité, la dangerosité, l'utilisabilité, l'accessibilité etc. L'utilité-valeur renvoie donc aux améliorations ou aux bénéfices significatifs que peut apporter l'artefact aux utilisateurs. Quelle que soit la méthode utilisée pour la mesurer, l'utilité-valeur caractérisée à un instant donnée couvre seulement une partie de l'étendue des possibles en termes de bénéfices, du fait de l'ensemble des combinatoires entre les propriétés et les situations potentielles, normales dégradées, conventionnelles, détournées, etc.

Inversement, l'utilité-destination renvoie explicitement à la description des fonctions du système (Nielsen, 1993) et des utilisations inscrites dans l'artefact. Cette description peut prendre des formes multiples et différentes en fonction du moment (concept, spécifications, prescription d'usage, maquette, prototype, artefact adaptés par l'utilisateur). L'utilitédestination correspond donc aux caractéristiques fonctionnelles et nonfonctionnelles de l'artefact. Il s'agit de fonctionnalités et de propriétés qui sont déterminées à un moment donné, même si elles peuvent être réaménagées et modifiées par la suite, y compris par les utilisateurs. L'utilité est à un moment donné " arrêtée " à partir non seulement d'une représentation sur des a priori possibles, mais aussi d'une représentation de ce qu'elle est concrètement face à une situation spécifique. Ainsi, Nielsen (1993) distingue, d'un côté, l'utilité désignant les buts que le système est censé permettre d'atteindre (" utility ", traduite par Tricot et al. (2003) au travers des termes "utilité théorique») et, de l'autre côté, l'utilité désignant le 
but que le système, utilisé par un utilisateur donné, permet effectivement d'atteindre dans des situations spécifiques ("usefulness ", c'est-à-dire l'utilité réelle / pratique ; Tricot et al. 2003). Cette dernière est considérée comme résultant de l'utilité théorique modulée par les propriétés d'utilisabilité de l'artefact considéré (voir figure 1).

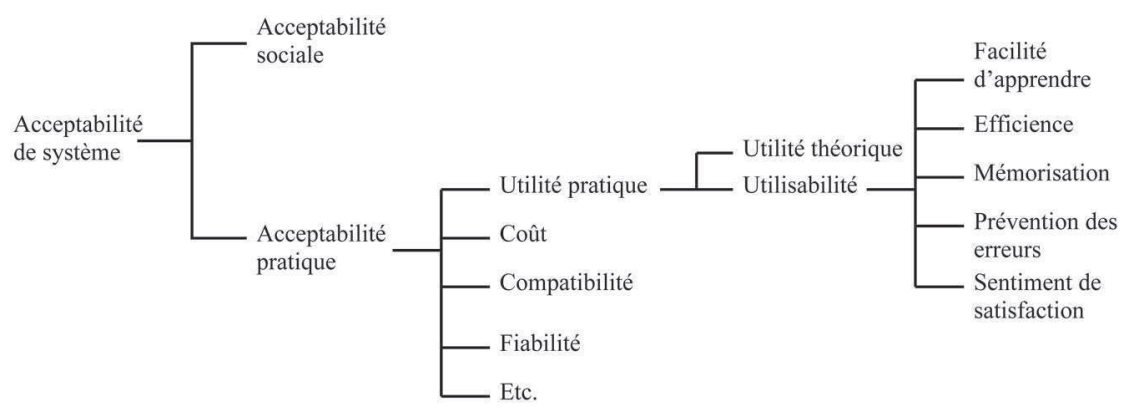

Figure 1: Modèle d'acceptabilité des systèmes de Nielsen (1993) selon la traduction de Tricot et al. (2003).

Figure 1: Nielsen's system acceptability model (1993) as translated by Tricot et al. (2003).

On soulignera qu'il n'y a aucun lien systématique entre les deux acceptions destination versus valeur. Certes, il est généralement souhaité que l'utilité (au sens de la destination) de l'objet conçu corresponde à une utilité (au sens de la plus-value) "réelle", tangible pour celles ou ceux qui vont l'utiliser. Néanmoins, tous les cas de figures sont envisageables, y compris celui où l'utilité-destination de l'artefact s'avérerait ne pas apporter une quelconque utilité-valeur à ses utilisateurs potentiels.

\section{I.2. UTILITÉ VERSUS BESOINS : DEUX FAÇONS DE CONSIDÉRER LES RELATIONS} ENTRE LES PROPRIÉTÉS DE L'ARTEFACT, LES UTILISATEURS ET LA SITUATION

Comme noté précédemment, les termes d'utilité et de besoin (ou des termes proches) traduisent le plus souvent dans la littérature un point de vue centré différemment sur l'un ou l'autre des éléments du triplet artefact/ utilisateur/situation. L'utilité, en tant que propriété de l'artefact particulier (ou d'une technologie si on généralise à une catégorie d'usages), caractérise ainsi le pôle "artefact" de la relation entre des sujets utilisateurs et un artefact (ou une classe d'artefact). Le pôle "utilisateur " de cette relation est souvent exprimé au travers de la notion de besoins, dans le sens où l'utilité renvoie chez plusieurs auteurs à la réponse (ou à l'absence de réponse) à un besoin. Il est par conséquent pertinent de considérer les liens qu'entretiennent les deux notions et les possibles complémentarités entre approches de l'utilité ou des besoins dans la conception.

La thématique de l'analyse du besoin en relation avec la conception d'artefacts innovants a donné lieu à des travaux récents en ergonomie. Ces travaux (Anastassova, 2006 ; Nicolas, 2000) montrent que la notion 
de "besoins" (" user needs " ou "requirements") est souvent mal définie. Elle n'est d'ailleurs pas partagée par l'ensemble des protagonistes de la conception, incluant les utilisateurs eux-mêmes. Dans son sens commun, un besoin est un manque de ce qui est perçu comme nécessaire (Le Petit Robert, 2007). Dans le domaine de la conception, un besoin est plutôt assimilé à une fonction ou un service associé à un produit, soit potentiellement (on parle alors plutôt de besoin au sens de "user needs "), soit de façon prescriptive (le terme "user requirement » est également traduit par le terme de "besoin"). Plusieurs typologies sont possibles pour tenter de préciser la notion. Trois principales se distinguent selon le critère utilisé pour décrire les besoins : leur degré d'explicitation et de réalisation à un instant donné, le contenu fonctionnel ou non, le niveau de généralité et d'échelle considéré.

\section{I.2.1. Degré explicite et réalisé des besoins}

La littérature considère trois types de "besoins " (Anastassova, 2006) selon leur nature explicite ou non, et selon qu'ils sont réalisés ou à venir :

Les besoins conscients. - Ils sont explicitement formulés par les (futurs) utilisateurs, c'est-à-dire "conscients " (Robertson, 2001). Les besoins conscients sont généralement incomplets, voire en décalage avec les besoins réels, et ce d'autant plus qu'ils sont assimilés sans précaution à la demande - laquelle provient rarement de l'utilisateur réel. Les besoins que le commanditaire est en capacité de prescrire et de formuler sont appelés " exigences " en génie industriel. Le terme de "besoins conscients" peut également correspondre partiellement à celui d'attentes exprimées par des clients potentiels dans le domaine du marketing. Une attente correspond à un souhait, un désir ou un idéal que le client exprime sans nécessairement s'attendre à ce qu'elle soit entièrement satisfaite, contrairement au besoin qui vise à être comblé.

Les besoins non conscients. - Ils résultent de plusieurs facteurs possibles (Robertson, 2001), L'absence d'opportunité offerte aux utilisateurs concernés pour réfléchir à leur activité actuelle est un premier facteur. Un second réside dans la méconnaissance qu'ont les utilisateurs des propriétés et des potentialités offertes par la technologie choisie, empêchant ainsi la mise en correspondance de ces potentialités avec les propriétés critiques de la tâche et de l'activité ciblée. Un autre facteur majeur réside dans la nature non verbalisable de certains besoins, liée notamment à une grande part d'automatismes dans l'activité. Certains automatismes peuvent d'ailleurs avoir été développés pour contourner ou minimiser des difficultés ou des manques quant à l'activité courante ;

Les besoins " latents 》. - Ils se caractérisent par leur nature non encore avérée ou encore "inimaginée " (" undreamed-of " requirements, Robertson, 2001). Ce troisième type de besoin (Sperandio, 2001) est un enjeu important pour les technologies émergentes, encore au stade du développement en laboratoire, et à la recherche de leurs applications. Or anticiper la technologie et les applications qui vont auront du succès constitue l'un des défis actuels de l'innovation intensive. 
Le lien entre utilité et ces types de besoins n'est toutefois pas univoque : le besoin peut désigner initialement les fonctions ou propriétés possibles, voire souhaitables pour l'artefact à concevoir ; ce besoin est ensuite traduit sous la forme de la spécification d'un sous-ensemble de fonctions et de propriétés de l'artefact sélectionnées. Puis une fois l'artefact concrètement réalisé, il devient alors possible de mesurer l'utilité effective au travers de l'usage et des utilisations. Vis-à-vis de l'utilité, le besoin peut constituer ainsi l'inspiration d'origine, l'argument et la justification associés à l'utilité d'un artefact au cours du processus de conception. La Figure 2 met en perspective les deux dimensions de l'utilité (destination et valeur) en regard des besoins des utilisateurs, exigences des commanditaires, des spécifications et des contraintes des concepteurs.

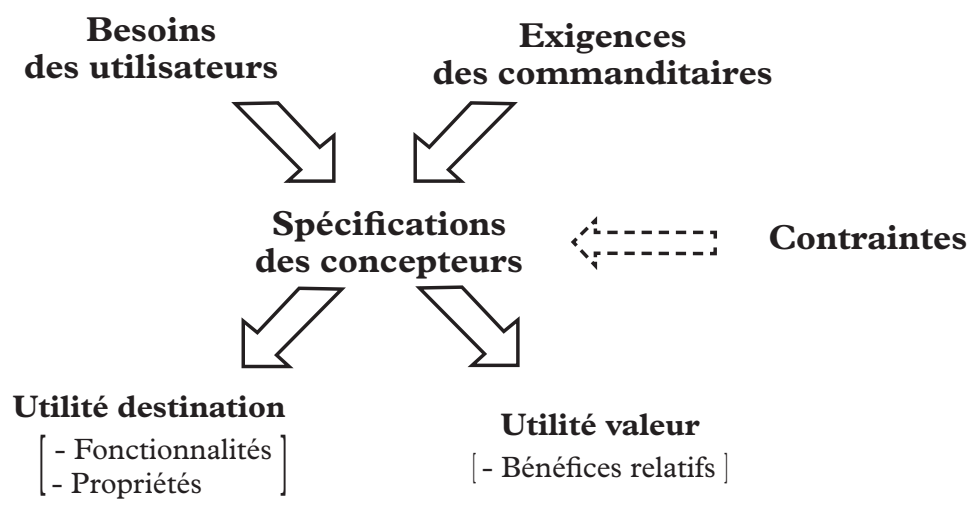

Figure 2 :Articulation entre utilité et termes connexes aux besoins.

Figure 2: Links between usefulness and words related to needs.

\section{I.2.2. Dimensions fonctionnelle et non fonctionnelle}

De façon orthogonale à la catégorisation précédente, deux classes de besoins sont également distinguées dans la littérature (Robertson, 2001). La première classe correspond aux "besoins fonctionnels ", c'est-à-dire aux fonctions qu'un système doit posséder, comme par exemple «enregistrer un film " ou "permettre de réaliser une action ». Les besoins fonctionnels représentent par conséquent les fonctions et les services avérés ou retenus du fait qu'ils sont jugés nécessaires ou avantageux pour les (futurs) utilisateurs.

La seconde classe correspond aux besoins non-fonctionnels, parfois qualifiés aussi d'hédoniques (Hornbæk, 200â). Il s'agit des qualités ou des attributs qu'un système doit avoir, comme le niveau de performance, la sécurité offerte, l'utilisabilité, la maintenabilité ou encore l'esthétique. Ces attributs correspondent à des qualités nécessaires ou avantageuses selon le point de vue des (futurs) utilisateurs. 


\section{I.2.3. Caractère spécifique ou général, individuel ou social}

Une autre question, peu abordée de façon systématique dans la littérature, concerne le caractère spécifique ou général d'un besoin, alors que cette caractéristique a des impacts sur les acteurs impliqués, sur les indicateurs et sur les méthodes permettant de prévoir et d'évaluer l'utilité. Le besoin, en tant que particularisation de la relation entre des utilisateurs et un artefact ou une technologie, peut s'analyser selon, d'une part, le caractère plus ou moins spécifique et des données considérées pour l'élaborer et d'autre part, selon les caractéristiques des utilisateurs ciblés.

Le besoin individuel. - Il est spécifique à un sujet particulier comme par exemple dans le cas d'un appareillage technologique lié à un handicap, ou dans le cas de la conception par l'utilisateur (" user-programming"); les besoins sont d'une certaine façon "incorporés " dans l'utilisateur ciblé, et leur prise en compte est a priori facilitée, à condition que l'utilisateur soit impliqué dans la conception de l'artefact et que des activités de communication entre les (futurs) utilisateurs et les concepteurs soient établies (Coughlan ï Macredie, 2002).

Le besoin construit en référence à une population spécifique. - Il s'agit du besoin associé à un groupe spécifique de sujets utilisateurs comme dans le contexte de la conception d'un outil propre à un métier, une expertise et une tâche donnés. Dans ce cas, les situations et les tâches de référence précises existent. Les utilisateurs constituent eux-mêmes une population spécifique et restreinte qu'il est possible d'impliquer en tant qu'acteurs de la conception. Cela favorise des interactions avec les concepteurs et facilite l'identification et l'évaluation des besoins et de l'utilité en termes de bénéfices potentiels que peut apporter l'artefact à l'utilisateur final.

Le besoin construit en référence à une population large et non spécifique. - Dans certains cas, la population présente des besoins, des caractéristiques et des compétences éminemment variables. Cela est par exemple le cas lors de la conception des dispositifs "grand public ». Contrairement à la situation précédente, les utilisateurs constituent une population plus large et plus hétérogène, d'une part, et les concepteurs de telles applications sont également des utilisateurs des artefacts qu'ils conçoivent, d'autre part. Les concepteurs adoptent à différents moments la posture d'un profil d'utilisateurs, ce qui est susceptible de limiter l'anticipation et l'évaluation des services et des fonctionnalités de l'artefact (utilité-destination). Toutefois, il est possible que les idées générées créent de nouveaux usages (approche "Technology Push "). Dans ces cas, les artefacts et leurs fonctionnalités sont issus de développements technologiques plutôt que de l'analyse des besoins. Il découle de cette hétérogénéité étendue trois difficultés. La première difficulté est que tous les utilisateurs n'ont pas les mêmes "besoins " en termes de fonctions, services et artefact détaillé (Kaiya, Shinbara, Kawano ï Saeki, 200囚). La deuxième difficulté est que pour chacun des besoins, les mesures d'avantages tirés diffèrent, voire sont hiérarchisés de manière différente. La troisième difficulté est que l'on ne peut généralement pas avoir à disposition toute la population d'utilisateurs. Cela implique que l'on travaille sur un échantillon seulement, ce qui pose la question de la définition exacte des frontières de la population de référence, de la 
construction de l'échantillon et de la représentativité des données qui en sont extraites, qu'il s'agisse d'idées "besoins " ou de mesure des bénéfices / avantages mesurés.

Au-delà de ces besoins liés à une utilisation individuelle (y compris dans le cas d'un nombre massif d'utilisateurs), un niveau différent peut être celui du besoin social, c'est-à-dire correspondant aux besoins exprimés au niveau de la société et des institutions ou de leurs émanations variées.

\section{I.2.4. Utilité et besoins évoluent et sont co-construits}

Les besoins, comme l'utilité, ne sont pas donnés a priori. Ils résultent d'une construction au cours des interactions entre les concepteurs et les utilisateurs. L'utilité réelle d'un système découle donc de la traduction pertinente des besoins des utilisateurs au niveau de l'artefact, sans toutefois s'y réduire pour au moins deux raisons. D'une part, les besoins sont susceptibles d'évoluer du fait de l'évolution du contexte et de l'apprentissage des utilisateurs. D'autre part, l'artefact et son environnement peuvent à leur tour révéler ou susciter des besoins inédits parmi les utilisateurs. Enfin, une question transversale concerne l'adaptation de l'artefact à d'autres utilisateurs que ceux n'ayant pas été impliqués dans la conception. Cette adaptation engendre des coûts non négligeables résultant de développements de nouvelles fonctionnalités nécessaires pour réduire le risque de non-acceptation.

\section{POINT DE VUE DE L'ERGONOMIE SUR L'UTILITÉ : UNE PROPRIÉTÉ CONSTRUITE ET ÉVOLUTIVE DES SYSTÈMES}

Le point de vue de l'ergonomie, et en particulier celui de l'ergonomie des activités de conception (Barcellini, Détienne \& Burkhardt, 2008 ; Blandford et al., 2007 ; Brangier \& Bastien, 2006 ; Burkhardt \& Sperandio, 2004) est donc que l'utilité de tout artefact n'est pas une propriété «donnée " mais construite et évolutive. L'utilité est une propriété construite car elle ne préexiste pas à l'objet conçu et émerge de l'interaction entre les acteurs concepteurs et/ou utilisateurs, des objets intermédiaires et des représentations diverses mobilisées autour de la conception. L'utilité est évolutive, c'est-à-dire qu'elle n'est ni complète ni définitive à chaque instant du processus de conception, mais change au cours du temps, en fonction de la façon dont changent les utilisateurs, l'artefact et l'environnement. Ainsi, l'utilité de l'artefact - ou le besoin recherché au travers de l'usage - évolue en parallèle des schèmes d'usage et des modifications éventuelles apportées par le sujet à l'artefact, voire aux modifications du contexte social et organisationnel dans lequel prend place l'utilisation (Bourmaud, 2007). 
Cette propriété dynamique fondamentale de l'utilité est cohérente avec les modèles actuels et les pratiques préconisées par la conception tels que la conception centrée sur les utilisateurs (Maguire, 2001), le développement par l'itération de phases de conception et d'évaluation (Bastien ï Scapin, 2004) et la conception continuée au travers de l'usage (Rabardel ï Béguin, 200â). L'utilité évolue en effet au gré des modifications de l'artefact, des changements dans l'environnement de travail mais aussi au fur et à mesure du temps et des connaissances que les utilisateurs élaborent. Anticiper et évaluer l'utilité tout au long du processus de conception est donc un enjeu fort. De même, il est souhaitable de développer une meilleure compréhension des liens entre l'utilité et d'autres critères de l'ergonomie, tels l'utilisabilité ou encore l'acceptabilité.

\section{II.1. L'UTILITÉ, UNE DIALECTIQUE CONTINUE ENTRE PROSPECTIVE ET ÉVALUATION RÉTROSPECTIVE}

La notion d'utilité, quelle que soit la facette considérée (c'est-à-dire destination versus valeur), renvoie à deux phases et à deux activités au cours de la conception. La première concerne la conception au sens de la définition progressive, itérative et de plus en plus précise de la destination du système au fur et à mesure du processus de conception, et de la valeur d'utilité dans les situations d'usages futures hypothétiques. La seconde correspond à l'évaluation, c'est-à-dire à la mesure plus ou moins précise de l'utilité associée à un état donné de la conception du système. Il en découle une démarcation entre deux univers de l'utilité d'un artefact, à chaque instant du processus de conception : un univers prospectif et un univers rétrospectif.

\section{II.1.1. Univers prospectif et univers rétrospectif}

L'univers prospectif de l'utilité correspond à l'univers des possibles envisagés par chaque acteur de la conception, relevant de la prédiction, de la projection ou encore de l'imagination des fonctions et de la forme de l'artefact - voire des futurs usages - avant leur réalisation. Cet univers prospectif est virtuel, hypothétique sans que les éléments qui le composent aient obligatoirement une cohérence intrinsèque et complète. Il peut être partiel, flou, conceptuel et peu partagé entre les acteurs de la conception à certains moments. Il peut inversement s'avérer à d'autres moments quasicomplet, précis et public, voire décrit en partie de façon formelle au travers de documents de spécifications de l'artefact à concevoir.

Il y a, par ailleurs, un univers rétrospectif de l'utilité, dans le sens d'une (re)construction des fonctions, de l'utilisation et des usages, sur la base de l'état actuel ou d'états précédents de l'artefact. Dans un sens restrictif centré sur l'artefact, cet univers pourrait être assimilé au résultat d'un processus de rétro-conception (retro-engineering) appliqué à l'artefact, ou de façon plus diffuse à l'application d'une démarche de réutilisation en génie logiciel (Burkhardt, 1997). Nous nous contenterons ici d'en souligner le caractère éminemment réducteur, dans la mesure 
où l'utilité implique de considérer le triplet utilisateurs/artefact/environnement et non la seule définition d'objectifs et de fonctions implantés dans un artefact. Dans le sens large où nous l'entendons, cet univers rétrospectif de l'utilité résulte de toutes les démarches qui visent à rendre explicite l'utilité, pour tout état particulier précédent de l'artefact, en tenant compte de l'état des connaissances de l'utilisateur et des propriétés de l'environnement dans lequel se déroule l'activité. Cet univers peut être issu d'une construction sociale mais aussi de la mise en œuvre de techniques empiriques visant la mesure et la validation de l'utilité, à un instant donné de la conception.

\section{II.1.2. Relations entre ces univers lors de la conception}

La conception implique une dialectique continuelle entre les deux univers depuis l'amont, où tant l'utilité que la forme et le détail de l'artefact restent flous et hypothétiques, jusqu'à l'aval où la forme finale est éprouvée et continue à évoluer dans l'usage. Pour faire simple, le premier univers regroupe l'ensemble des particularités, besoins ou solutions pensables (mais pas forcément souhaitables), tandis que le second univers est assimilable à l'ensemble des expériences pertinentes en termes d'utilité pour informer la conception de l'artefact. La mise en exergue de ces deux univers provient de l'idée que l'utilité effective d'un artefact est en grande partie déterminée par la façon dont ces deux univers sont explorés de manière approfondie et articulée par les acteurs de la conception. Ne pas construire et explorer l'un ou l'autre de ces deux univers, ou encore le faire de façon partielle ou superficielle, comporte en effet le risque de définir un artefact apportant aucune, sinon peu, d'amélioration de l'existant en ce qui concerne les contraintes, les défaillances observables et l'assistance aux objectifs poursuivis par les utilisateurs. Quand bien même il y a amélioration, un autre risque est que cette amélioration porte sur des aspects fonctionnels ou nonfonctionnels de faible valeur pour les utilisateurs. Il se peut par ailleurs que l'utilité-destination de l'artefact ne corresponde à aucun objectif ou besoin des utilisateurs, engendrant dans le meilleur des cas, la création d'un nouvel usage, sans pour autant répondre nécessairement aux besoins qui préexistent.

Les modèles et travaux empiriques sur l'activité de conception reconnaissent son caractère fondamentalement itératif et/ou opportuniste (Boehm, 1986 ; Tudhope, Beynon-Davies \& Mackay, 2000 ; Visser, 1994), caractère d'ailleurs mis en exergue dans les approches récentes (Agile, XP - Extreme Programming). Dans une approche centrée sur les utilisateurs, les itérations impliquent la participation des utilisateurs tant sous l'angle de la contribution aux spécifications que sous l'angle de l'évaluation des propriétés de l'artefact.

La Figure 3 formalise les relations entre la construction des deux univers, l'utilité potentielle et l'utilité réelle. Ce modèle doit être vu comme un processus itératif et continu, dans lequel le contenu des deux univers change dynamiquement avec le temps et les acteurs de la conception. 


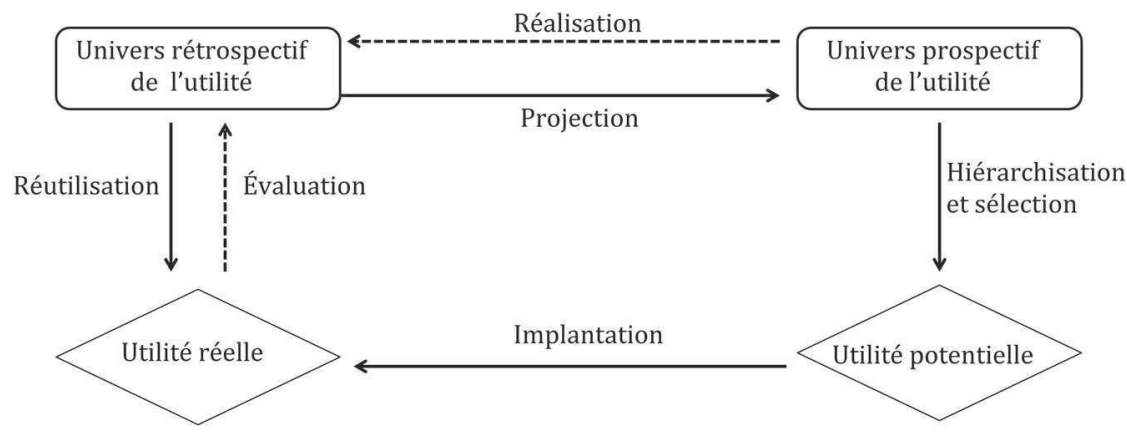

Figure 3 : Relations entre univers prospectif et rétrospectif de l'utilité, utilité potentielle et utilité réelle.

Figure 3: Relations between prospective and retrospective worlds, potential and effective usefulness.

Les acteurs de la conception (c'est-à-dire utilisateurs et concepteurs) projettent les besoins, qui découlent de l'univers rétrospectif, vers l'univers prospectif. En d'autres termes, à partir des besoins intrinsèques à l'univers rétrospectif, les participants infèrent d'autres besoins (c'est-à-dire l'univers prospectif). Le passage de l'univers prospectif (c'est-à-dire le champ de tous les possibles) vers l'utilité potentielle se caractérise par le choix des fonctions et formes possibles de l'artefact retenues.

\section{II.1.3. Rôle des utilisateurs dans le processus de conception}

Au sein du processus de conception, tous les participants n'ont pas les mêmes capacités d'action en regard de leur expertise technologique et des finalités attendues de l'artefact, ni une possibilité similaire et équilibrée de participation aux discussions de conception. La mobilisation effective et l'articulation respective des deux univers rétrospectif et prospectif de l'utilité varient ainsi selon la place plus ou moins grande laissée aux utilisateurs, ainsi que selon la mise en place ou non d'outils de facilitation pour un apprentissage par échanges mutuels communs (Béguin, 2003). Lorsque la participation et l'expérience des utilisateurs sont peu sollicitées, l'univers prospectif est privilégié, avec peu de considération pour l'univers rétrospectif - d'autant que l'on se situe dans un contexte d'innovation technologique forte (typiquement, il est affirmé que telle technologie est tellement innovante que cela ne sert à rien de regarder l'existant et l'activité actuelle des (futurs) utilisateurs car tout va changer !). Dans une approche centréeutilisateur, l'univers rétrospectif est habituellement plus mobilisé au travers de méthodes visant à extraire l'expérience des utilisateurs ainsi que par le moyen d'itération de tests et évaluations. L'univers prospectif de l'utilité reste en grande partie celui des concepteurs, même s'il correspond concrètement à une interprétation des demandes et des attentes recueillies auprès des (futurs) utilisateurs. Enfin dans une approche participative, il y a d'une 
part l'idée de co-construction des deux univers impliquant une coopération et des apprentissages croisés entre concepteurs et utilisateurs et, d'autre part, la participation des utilisateurs aux processus de décision concernant la définition de l'utilité ciblée au travers de la conception de l'artefact.

\section{II.2. L'UTILITÉ ET LES AUTRES CRITÈRES DE L'ERGONOMIE}

\section{II.2.1. Utilité et utilisabilité}

L'utilité et l'utilisabilité sont deux critères qui ne se confondent pas. L'utilisabilité correspond à la mesure (ou à une estimation probable) de la performance des utilisateurs, dans un contexte et pour une utilisation prévue $^{1}$. Or un produit informatique peut être très satisfaisant sur le plan de l'utilisabilité, sans pour autant permettre à l'utilisateur de satisfaire convenablement ses buts. Comme nous l'avons mis en exergue précédemment, l'utilité, par opposition, qualifie le degré d'adéquation ou de coïncidence entre les buts poursuivis par les utilisateurs et ceux rendus possibles par l'outil considéré comme un moyen parmi - et en coordination avec - d'autres éléments du contexte d'activité. Ainsi, alors que l'utilisabilité correspond à une ou plusieurs mesures de la performance des utilisateurs manipulant l'artefact pour atteindre un sous-ensemble spécifié de buts, la dimension de l'utilité concerne la propriété de pertinence ou encore d'adéquation caractérisant le sous-ensemble des buts spécifiés euxmêmes, c'est-à-dire les buts qu'il est souhaitable ou requis de permettre de réaliser avec le système en cours de conception. De ce fait, l'utilisabilité peut interférer de façon négative avec l'utilité dans le sens où l'utilisabilité peut diminuer la probabilité d'accès à la fonction intéressante pour le sujet utilisateur, par exemple du fait de la complexité de mise en œuvre des procédures d'utilisation, de choix et d'une organisation arbitraires des objets de l'interface, etc. Cette position de Nielsen a toutefois été critiquée (Tricot et al., 2003) en ce que le choix et le degré d'adéquation des fonctions peuvent être aussi à l'origine des difficultés de l'utilisateur, toutes les difficultés ne se réduisant pas à un problème d'utilisabilité ${ }^{2}$ dans le sens d'une restriction de l'accès des utilisateurs à des fonctions réputées utiles pour leurs objectifs. Tricot illustre son propos dans le domaine de la recherche d'informations au travers d'un moteur de recherche. Il montre que l'utilité comparée de moteurs différents est essentiellement liée à la pertinence et à l'intérêt des informations trouvées par le sujet, et non la seule résultante d'un problème d'utilisabilité. En d'autres termes, ne pas accéder à une information donnée provient soit d'un problème d'utilisabilité, soit du fait de l'absence de l'information recherchée elle-même et donc de l'absence d'utilité du système. L'utilisabilité peut également interférer de façon positive, en suscitant des procédures différentes, voire en conduisant à la construction de nouveaux besoins et de nouvelles connaissances.

1. Cf. Définition Norme ISO 9241-11.

2. Un défaut d'utilisabilité peut aussi éventuellement interférer de façon positive, en suscitant des procédures différentes, voire en conduisant à la construction de nouveaux besoins et de nouvelles connaissances. 
En ce sens, l'utilisabilité est une caractéristique de l'outil qui facilite plus ou moins les actions des utilisateurs qui "rendent concrètes " les propriétés et les fonctionnalités intrinsèques de l'artefact (utilité-destination) dans une situation donnée.

\section{II.2.2. Utilité et acceptabilité}

Nielsen (1993) distingue deux catégories d'acceptabilité : l'acceptabilité pratique et l'acceptabilité sociale.

L'acceptabilité pratique fait référence à des modèles d'ergonomie cognitive (par exemple, utilisabilité, modèles de la tâche, modèles de l'utilisateur). En ce sens, l'acceptabilité est déterminée par le niveau d'utilisabilité et le niveau d'utilité de l'artefact pour l'utilisateur en tant qu'individu cognitif. L'utilité est ici définie par l'adéquation entre les fonctions de l'artefact technologique (c'est-à-dire utilité-destination) d'une part, et les buts des utilisateurs ainsi que les contextes d'usages (c'est-à-dire utilitévaleur) d'autre part (Wolff, Burkhardt i De La Garza, 200囚). Cette détermination met en évidence qu'un défaut d'acceptabilité peut apparaître dans deux cas. Dans le premier cas, le système bien que facile à utiliser n'a pas un niveau d'utilité suffisant pour être accepté par l'utilisateur. Dans le second cas, le système, bien qu'utile à l'opérateur, n'a pas un niveau d'utilisabilité suffisant.

L'acceptabilité sociale fait plutôt référence à des modèles psychosociaux (par exemple, Technology Acceptance Model ou TAM, Figure 4). L'acceptabilité est déterminée par les représentations de l'utilité et de l'utilisabilité de l'artefact par l'utilisateur en tant qu'individu social. En ce sens, les perceptions de l'utilité et de l'utilisabilité dépendent des interprétations faites par l'individu concernant les contextes sociaux et technologiques, et les transformations organisationnelles engendrées par ces interprétations afin de favoriser l'usage des artefacts.

Brangier, Hammes-Adelé et Bastien (2010) ne se satisfaisant pas des deux approches précédentes de l'acceptabilité, ont proposé une approche alternative fondée sur l'idée de la symbiose entre humain-technologieorganisation. Cette approche repose sur l'idée que la technologie est un prolongement de l'humain et que les deux entretiennent une relation de changements réciproques continus. Les auteurs posent comme principe que l'usage de la technologie dépend de trois déterminants de la symbiose humain-technologie-organisation : les fonctionnalités (c'est-à-dire utilitédestination), l'utilisabilité de la technologie et les formes de régulation liées aux comportements socio-organisationnels, par exemple, appropriation, résistance, innovation sociale, etc. Ces comportements révèlent un agrément plus ou moins significatif de la technologie pour les individus (c'est-à-dire utilité-valeur).

Finalement, l'acceptabilité est la perception que l'utilisateur a de l'utilité-valeur a posteriori, c'est-à-dire des bénéfices associés aux propriétés intrinsèques de l'artefact en regard de la situation d'usage. Ainsi, un artefact ne sera pas accepté s'il est inutile ou inutilisé à cause d'un défaut d'utilité-valeur. 


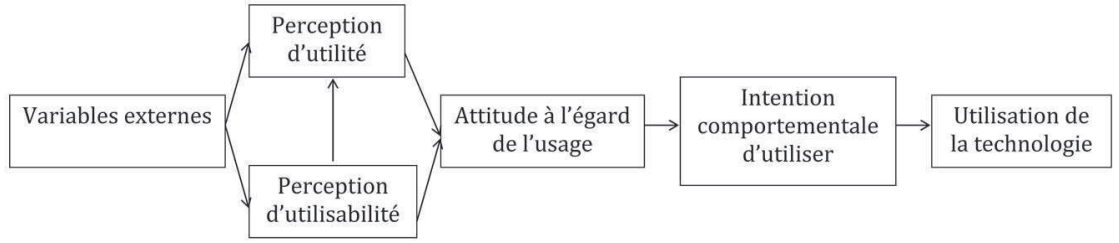

Figure 4 : Modèle d'acceptabilité de technologie (Davis, 1989), traduit par Brangier (2009).

Figure 4:Technology Acceptance Model (1989), translated by Brangier (2009).

\section{II.2.3. La créativité comme source pour l'utilité et l'innovation}

Un paradoxe déjà mentionné est que l'existant, tant du point de vue technique que des compétences, connaissances et savoir-faire actuels des personnes concernées par l'informatisation, constitue la base privilégiée pour fonder les spécifications et les préconisations ergonomiques en matière de besoin et de sélection des fonctions et des propriétés souhaitables en termes d'utilité au cours de la conception.

Il existe peu d'études, dans la littérature sur la conception, s'intéressant au lien entre l'utilité et les approches ou les méthodes favorisant la créativité, voire l'innovation (Mich, Anesi ï Berry, 2004 ; Nguyen ï Shanks, 2009). En général, les travaux qui s'intéressent à l'utilité font référence à un modèle des objectifs à atteindre pour assister l'utilisateur (Diez i McIntosh, 2009 ; Scholtz et al., 2006). Ceux qui traitent d'approches ou de méthodes favorisant la créativité, voire l'innovation, tendraient plutôt à éviter de raisonner en termes d'utilité afin d'ouvrir l'espace des possibles et d'apporter des propositions ou solutions inédites (Johnson $\ddot{i}$ Carruthers, 2006 ; Bonnardel ï Marmèche, 200â ; Sternberg, 200â).

\section{UNE SYNTHĖSE DES MÉTHODES \\ ET DE LEURS CONTRIBUTIONS \\ AUX DIFFÉRENTES DIMENSIONS DE L'UTILITÉ : VERS UNE CONCEPTION POUR L'UTILITÉ}

\section{III.1. CONCEVOIR POUR L'UTILITÉ}

Sous l'angle de la conception pour l'utilité, le processus de conception implique trois phases qui ne supposent pas forcément un découpage séquentiel strict dans le processus de conception (Tableau 1). La première est la production des "besoins" et des spécifications candidates, ainsi que la génération des spécifications au travers de processus créatifs. La deuxième concerne la prise de décision en regard de cet éventail de possibles. La troisième renvoie à la mesure et l'évaluation de l'utilité associée spécifiquement à l'utilisation d'un existant en situation. 
Tableau 1 - Dimensions de l'utilité et phases de l'activité au coup de la conception.

Table 1 - Usefulness in design and phases in design activity.

\begin{tabular}{|l|l|l|l|}
\cline { 2 - 4 } \multicolumn{1}{c|}{} & \multicolumn{1}{c|}{$\begin{array}{c}\text { Univers } \\
\text { prospectif }\end{array}$} & \multicolumn{1}{c|}{$\begin{array}{c}\text { Décision } \\
\text { et Implémentation }\end{array}$} & \multicolumn{1}{c|}{$\begin{array}{c}\text { Univers } \\
\text { rétrospectif }\end{array}$} \\
\hline $\begin{array}{l}\text { Utilité- } \\
\text { destination }\end{array}$ & $\begin{array}{l}\text { Production d'hypo- } \\
\text { thèses sur les fonc- } \\
\text { tions, les services, } \\
\text { les dialogues et les } \\
\text { propriétés non- } \\
\text { fonctionnelles }\end{array}$ & $\begin{array}{l}\text { Hiérarchisation et } \\
\text { décisions quant aux } \\
\text { fonctions, services, } \\
\text { dialogues et interfaces } \\
\text { à développer }\end{array}$ & $\begin{array}{l}\text { Mesure et évalua- } \\
\text { tion de la perti- } \\
\text { nence des choix, } \\
\text { identification des } \\
\text { défaillances }\end{array}$ \\
\hline $\begin{array}{l}\text { Utilité- } \\
\text { valeur }\end{array}$ & $\begin{array}{l}\text { Production d'hypo- } \\
\text { thèses sur les } \\
\text { bénéfices quanti- } \\
\text { tatifs et qualitatifs } \\
\text { attendus }\end{array}$ & $\begin{array}{l}\text { Sélection et opéra- } \\
\text { tionnalisation des } \\
\text { hypothèses privilégiées } \\
\text { en termes de bénéfices } \\
\text { et d'avantages }\end{array}$ & $\begin{array}{l}\text { Mesure et évalua- } \\
\text { tion des bénéfices } \\
\text { et des avantages }\end{array}$ \\
\hline
\end{tabular}

Dans la suite de cette section, nous proposons une synthèse des contributions de différentes méthodes au développement d'une conception pour l'utilité. Les méthodes exposées ne représentent qu'une partie des méthodes disponibles. Nous avons fait le choix de citer des méthodes qui illustrent une complémentarité entre les différentes perspectives en termes d'utilité comme réponse à des besoins :

- L'utilité est une réponse à des besoins conscients accessibles par des entretiens ;

- L'utilité est une réponse à des besoins avérés mais non conscients chez les utilisateurs, que l'on peut identifier au moyen d'observations ;

- L'utilité est une réponse à des besoins latents susceptibles de se construire lors d'allo-confrontations.

\section{III.2. Production D’HYPOTHÈSES ET ÉlABORATION D’UNIVERS PROSPECTIFS}

La formulation d'hypothèses sur les bénéfices attendus implique une identification claire des destinataires, de la nature et de l'ampleur des avantages ciblés, cela pour chacun des différents contextes d'usage anticipés. L'utilité, telle qu'elle pourra être mesurée au final, est déterminée en partie par cette étape de production puis de sélection d'hypothèses sur les avantages à cibler.

\section{III.2.1. Les besoins et l'activité comme sources de construction d'hypothèses}

Pour permettre d'accéder à l'utilité dans le cas de l'univers prospectif, il est nécessaire de comprendre la nature des tâches et des processus conscients et inconscients de l'activité de référence (c'est-à-dire celle dans laquelle l'artefact sera intégré). Toutefois, s'il y a un manque d'intérêt 
(ou un intérêt trop tardif) pour la compréhension de la nature des tâches des utilisateurs potentiels, cela peut engendrer des "défauts d'utilité " (Burkhardt, 2006). Il existe des modèles et des méthodes d'analyse des besoins qui permettent de repérer les besoins actuels ou potentiels relativement à l'activité actuelle.

L'analyse des difficultés lors de la réalisation de l'activité existante (à savoir la situation n'intégrant pas l'artefact à concevoir) et la mise en évidence des déterminants expliquant ces difficultés sont des supports pour l'élaboration d'hypothèses sur les plus-values que pourrait apporter l'artefact par rapport à la situation existante. Identifier les plus-values réellement avantageuses permet ensuite de proposer un système qui ait une bonne utilité-valeur a priori pour les utilisateurs. Sur le plan méthodologique, les entretiens permettent de recueillir un certain nombre de difficultés et de manques dont les utilisateurs ont conscience au sein de la situation existante (Anastassova, Burkhardt, Mégard \& Leservot, 200区). Complémentairement, les observations complètent permettent d'une part d'accéder à d'autres difficultés et manques dont les utilisateurs n'avaient pas conscience ou qu'ils ne parvenaient pas à verbaliser. Les observations permettent d'autre part d'identifier des facteurs explicatifs à ces difficultés (Anastassova et al., 200区).

La variabilité est inhérente à l'activité, et l'ergonomie possède des méthodes pour étudier la variabilité intra-individuelle, la variabilité interindividuelle, la variabilité des contextes, la variabilité des contraintes et la variabilité des pratiques. Par exemple, Mollo et Falzon (2004) ont montré que les allo-confrontations permettaient de produire des données sur la variabilité des pratiques. L'analyse des facteurs de variabilité dans l'activité existante est un support qui permet de formuler un large spectre d'hypothèses sur les avantages de l'artefact pour les utilisateurs. La connaissance des sources de variabilité contribue à enrichir l'utilité au sens de la valeur.

Les données récoltées sur l'activité principalement à partir d'entretiens, d'observations ou d'auto- confrontations et d'allo-confrontations sont formalisées dans des modèles de l'activité ou des modèles de tâches (Couix, 2007). Ces formalisations décrivent la compréhension détaillée des tâches des utilisateurs (ordonnancement des tâches, fréquence, difficultés, etc.), de leur environnement et de leurs contraintes (Burkhardt, 2006). Elles sont un bon outil pour déterminer l'organisation des éléments dans l'interface, ou bien les caractéristiques non-fonctionnelles de l'artefact. Ces modèles sont un moyen de passer progressivement d'une analyse de l'activité à une inscription des besoins en termes de fonctionnalités (Haradji \& Faveaux, 2006) et de concevoir des artefacts pour lesquels l'utilité-destination correspond a priori à l'utilité-valeur.

\section{III.2.2. L'imagination créative comme source de construction d'hypothèses}

Il existe plusieurs modèles et méthodes d'anticipation ou d'accompagnement au développement d'utilités possibles (c'est-à-dire usages et valeurs associées). Le terme créatif est ici entendu au sens d'invention de 
nouvelles utilisations de l'outil et usages "non contenus " ou non directement déductibles de l'existant (c'est-à-dire des besoins actuels identifiés).

Pour générer des idées de concepts de nouveaux produits ou anticiper des usages potentiels d'un artefact, le marketing emploie, entre autres, la méthode prospective. Cette dernière a pour objectif de :

- concevoir et représenter les mutations et les formes possibles de produits (prospective produit, prospective technologique) et d'organisation socio-économique d'une société ou d'un secteur d'activité dans un avenir plus ou moins éloigné (prospective économique, prospective territoriale) ;

- définir des choix et des objectifs à long terme pour les prévisions à court ou moyen terme (Trésor de la Langue Française, n.d.).

Pour concrétiser cette méthode, considérons succinctement un exemple d'étude prospective (Jolly et al., 2008). L'objectif de cette étude, impliquant dix spécialistes de domaines différents et complémentaires, était de fournir des axes d'innovation vers lesquels les entreprises devraient, selon eux, évoluer. La méthode employée est de décrypter les évolutions de la société. Par exemple, pour des enfants ne vivant pas avec leurs deux parents (sous le même toit), une contrainte importante est de transporter sans cesse leurs affaires scolaires. Une solution concrète exposée a été le cartable électronique qui permettrait aux enfants de disposer de tout leur matériel scolaire en tout lieu. Cette étude montre qu'en prospective, l'utilité comprise au sens de la destination est abordée sous l'angle d'hypothèses sur les concepts (par exemple, concept du cartable électronique), les fonctions (par exemple, accès aux bases de données de matériels scolaires) ou les usages (par exemple, possibilité de faire ses devoirs dans la voiture). La prospective contribue à imaginer de nouveaux usages sans pour autant tenter de détailler l'activité future. Il s'agirait de prévoir différentes formes possibles d'activité (Daniellou, 1992) plutôt que de s'enferrer dans une seule hypothèse. Le large spectre d'usages ou de services contribue à enrichir l'utilité au sens de la destination.

\section{III.2.3. L'évaluation et les données issues de l'univers rétrospectif comme sources de construction d'hypothèses}

La mise à l'épreuve de l'utilité, au travers de la réalisation et de la mise en situation, engendre une réflexion sur le besoin, voire le pousse à évoluer. Concrètement, il existe des méthodes permettant de faire émerger de nouveaux besoins et de les intégrer au processus de conception. Cette partie fait notamment référence au rôle de l'évaluation d'objets intermédiaires (c'est-à-dire prototype, maquette, etc.) sur l'identification des besoins et la construction de l'utilité.

L'évaluation de prototypes est une méthode de simulation au sens d'une reproduction artificielle d'un phénomène à étudier visant la reconstitution d'une situation de travail ou d'utilisation afin d'observer et d'induire des comportements proches de ceux susceptibles d'apparaître 
dans une situation réelle. Les techniques de simulation s'inscrivent dans une démarche de conception des technologies innovantes reposant sur le prototypage rapide (Brangier i Bastien, 2006). Les prototypes sont des supports à la compréhension des concepts technologiques flous pour ceux qui n'ont pas une expertise technologique suffisante (ergonomes, futurs utilisateurs, managers, designers, etc.) en aidant les opérateurs à développer les ressources de leur action et en leur permettant de développer les conceptualisations dont ils ont besoin pour en parler avec les concepteurs (Anastassova, Burkhardt ï Mégard, 2007a). Cette compréhension du système et de ses contraintes technologiques par les utilisateurs fait émerger de nouveaux besoins au sens de l'utilité-destination (c'est-à-dire de nouvelles fonctions). Les prototypes sont également un moyen pour projeter les utilisateurs dans un contexte d'usage potentiellement futur intégrant l'artefact en cours de conception. Les utilisateurs sont alors en capacité de proposer des fonctions de l'application future jusque-là latentes et nonconscientes (Sperandio, 2001), et contribuent de fait à améliorer l'utilitédestination en intégrant ces nouveaux besoins au cours de la conception (Newell, Carmichael, Morgan ï Dickinson, 2006). Les utilisateurs sont également capables de se projeter dans la situation future incluant l'artefact et d'en mesurer les avantages ou les bénéfices potentiels par rapport à la situation existante. Aussi, l'évaluation de prototype est un moyen d'enrichir l'utilité-valeur. Dans ce double objectif, les travaux suggèrent d'ailleurs de confronter les utilisateurs à plusieurs solutions très différentes plutôt qu'à une seule (Tohidi, Buxton, Baecker ï Sellen 2006).

La méthode CASSM, ou Concept-based Analysis of Surface and Structural Misfits (Blandford, Connell ï Green, 2004) est une méthode d'analyse des inadéquations entre les "concepts utilisateurs " (c'est-à-dire les informations sur la manière selon laquelle est utilisé le système dans son contexte d'usage normal) et les "concepts système " (c'est-à-dire les informations sur l'ensemble du système). Il s'agit donc d'identifier les adéquations (Wolff et al., 200â) ou les discordances (Blandford et al., 2007) entre l'utilité au sens de la destination de l'artefact (c'est-à-dire les fonctions et performances du système) et l'utilité au sens de la valeur pour l'utilisateur (c'est-à-dire les buts des utilisateurs et contextes d'usages), afin de proposer de nouvelles fonctions ou de nouvelles qualités non-fonctionnelles de l'artefact et, de fait, de rectifier l'utilité-destination du système.

\section{III.3. HiÉRARCHISATION, SÉLECTION ET PRISE DE DÉCISIONS}

Le passage de l'univers prospectif à l'utilité potentielle réside dans le choix des besoins qui sont choisis pour être pourvus dans le projet développé.

III.3.1. La sélection des fonctions par les concepteurs suivie de leur hiérarchisation par les utilisateurs

Dans le cadre de certains projets de conception de systèmes innovants (Loup-Escande, Burkhardt, Christofol, $\ddot{i}$ Richir, 2010), il n'est pas rare que les concepteurs demandent à un panel de personnes représentant 
les "utilisateurs " de hiérarchiser une liste de besoins présélectionnés par les ingénieurs eux-mêmes, selon les contraintes techniques ou de coût. Le terme "d'utilisateur " est cependant souvent assimilé au représentant de "l'entreprise " ou au commanditaire, plutôt qu'aux personnes concrètement concernées par l'artefact à concevoir, c'est-à-dire les utilisateurs finaux au sens de l'ergonomie. Le risque de la sélection des fonctions par les concepteurs eux-mêmes en amont de la hiérarchisation par les utilisateurs est que l'artefact n'apporte pas de réelle valeur ajoutée aux utilisateurs dans leur activité. En effet, la fonctionnalité implémentée n'apporte pas systématiquement un avantage ou un bénéfice à l'utilisateur final, puisque le besoin identifié et considéré comme le point de départ du processus de conception provient du concepteur lui-même ou du commanditaire.

\section{III.3.2. La hiérarchisation et la sélection des fonctions par le groupe concepteurs-utilisateurs}

Dans d'autres projets de conception (Plos, Buisine, Aoussat \& Dumas, 2007), la hiérarchisation et la sélection des fonctions sont réalisées par un groupe de travail constitué de concepteurs et d'utilisateurs. L'avantage d'intégrer des utilisateurs au niveau de la sélection des fonctions est de concevoir un artefact pour lequel l'utilité-destination correspond à l'utilitévaleur pour l'utilisateur. En d'autres termes, les propriétés «formelles » de l'artefact (c'est-à-dire ses fonctionnalités et ses caractéristiques) utilisées représentent une plus-value ou une vertu réelles pour les utilisateurs.

\section{III.4. ÉVALUER L'UTILITÉ}

Pour évaluer l'utilité, l'ergonomie dispose de critères et de méthodes spécifiquement adaptés selon que l'on évalue l'utilité-destination ou l'utilitévaleur.

\section{III.4.1. Les critères d'évaluation}

L'évaluation des choix relatifs aux fonctions, à leur spécification détaillée en termes de dialogues et d'interfaces puis à leur implémentation implique l'élaboration de critères pour mesurer la pertinence des "besoins " identifiés grâce aux différentes méthodes (par exemple, entretiens, observations, etc.).

En psychologie et en ergonomie, l'utilité d'une application au sens de la valeur se mesure selon des modalités subjectives comme l'agrément et la performance perçue (Davis, 1989), mais aussi selon des critères objectifs comme l'efficacité, le coût, la rapidité d'atteinte des objectifs voulus dans une situation écologique. Dans le domaine de l'apprentissage, la qualité du transfert permet d'évaluer l'efficacité de l'apprentissage et l'utilité du système d'aide à l'apprentissage (Anastassova, Burkhardt, Megaro \& Ehanno, 2007b). En marketing, certains auteurs ont une 
vision très réductrice de l'utilité d'un produit, qui est pour eux assurée dès lors qu'il existe une intention d'achat par des utilisateurs potentiels (Premkumar i Bhattacherjeeb, 2006). Or, le lien entre intention d'achat, achat réel et utilisation effective est loin d'être évident et semble trop simpliste. En effet, ce n'est pas parce que le consommateur exprime une intention d'achat qu'il achètera effectivement l'artefact ; rien ne permet d'affirmer non plus que s'il l'achète, il l'utilisera effectivement. Pour Rothwell (1994), c'est l'utilité d'un objet conçu qui détermine son succès commercial. Cela ne permet pas d'anticiper ou d'évaluer les bénéfices associés au produit.

\section{III.4.2. L'inspection de l'artefact}

L'identification des défaillances implique des évaluations itératives, aussi bien au cours de la conception du dispositif qu'une fois le système en usage. Par exemple, les méthodes d'inspection consistent en une analyse exhaustive de l'ensemble du logiciel et un examen de l'interface par un concepteur (Nielsen ï Mack, 1994 ; Virzi, 1997 ; Baccino, Bellino i Colombi, 200â ; Cockton, Woolrych ï Lavery, 2008). Cet examen se fait au regard des spécifications dont l'adéquation aux besoins fonctionnels et non-fonctionnels des utilisateurs dépend en grande partie des méthodes d'analyse et des acteurs impliqués dans leur énoncé a priori. Or ce type d'évaluation est fondé sur la représentation d'un tiers's, qui n'est pas l'utilisateur final. À force de réaliser des inspections hors situation réelle de travail, le concepteur se fait une idée fausse ou partielle de la tखche et se construit sa propre représentation qui n'est pas celle souhaitée par les utilisateurs (Darses ï Wolff, 2006). Le risque est que si les spécifications sont initialement peu adaptées en termes d'utilité au sens de la destination, alors l'évaluation pourra conclure que les fonctions sont pertinentes sans que l'artefact final soit utile au sens de la valeur. Ce type de méthode permet essentiellement de valider la réalisation ou non des spécifications, ainsi que d'identifier les principaux problèmes d'utilisabilité et leurs solutions possibles.

\section{III.4.3. L'évaluation des défauts d'utilité}

L'utilité des propriétés intrinsèques d'un artefact peut être évaluée à des instants différents de la vie du processus de conception (c'est-à-dire évaluation d'un prototype hautement fidèle, évaluation du produit final avant utilisation en entreprise) afin d'en mesurer la "valeur " associée pour l'utilisateur ou le groupe d'utilisateurs. Cette mesure peut s'effectuer sur le moyen terme, comme sur le long terme (c'est-à-dire évaluation en entreprise lorsque le système est en cours d'utilisation). Par exemple, (Gaudart, 2000) a réalisé une étude longitudinale pour évaluer l'utilité d'un logiciel. Elle a montré qu'une fonction a priori utile au sens de la destination (par

1. Ergonomes ou ingénieurs ayant a priori une meilleure connaissance des technologies à mettre en œuvre que des utilisateurs réels. 
exemple, espace de dialogue) répondait peu aux besoins des utilisateurs notamment à cause notamment des difficultés d'accès, et possédait de fait une faible utilité-valeur. L'auteur a également montré que les utilisateurs utilisaient d'autres espaces jugés plus utiles au sens de la valeur, malgré la nécessité de mettre en place de nouvelles règles d'usage (par exemple, abréviations, informations pertinentes).

\section{III.5. REPRÉSENTATIONS ET ÉCHANGES MUTUELS ENTRE ACTEURS}

L'utilité-valeur est plus facile à construire par les utilisateurs sur la base de leur expérience, dans une approche rétrospective. En effet, ce qui permet aux utilisateurs d'établir des hypothèses sur les bénéfices potentiels d'un artefact est a minima une bonne représentation des caractéristiques de leur activité existante (c'est-à-dire sans l'artefact) couplée à une connaissance suffisante du prototype de l'artefact, et idéalement une insertion de l'artefact dans la future activité potentielle. Inversement, il n'est pas si difficile pour des concepteurs de proposer des fonctions (c'est-à-dire utilité-destination) sur la base de leur propre expérience de concepteur car ils ont une bonne connaissance de la technologie. Dans ce contexte, l'expérience du concepteur lui sert à la définition de l'univers prospectif, sans qu'il n'y ait systématiquement d'ancrage réel dans l'univers rétrospectif des utilisateurs. Ainsi, il n'est pas rare que les fonctions "utiles " a priori au sens de la destination répondent peu aux besoins réels des utilisateurs a posteriori (c'est-à-dire faible utilité-valeur), voire soient justifiées par d'autres motifs comme l'analyse concurrentielle, la compatibilité avec d'autres lignes de produits, les caractéristiques offertes par les composants disponibles, etc.

Des méthodes telles que les "persona " et les " use cases " permettent de faciliter la communication et les échanges entre utilisateurs et concepteurs. Ceci permet aux concepteurs de concrétiser et de se représenter les besoins des utilisateurs dans une logique prospective.

L'appartenance des besoins aux univers prospectif ou rétrospectif dépend du caractère plus ou moins conscient des besoins considérés. Lorsque les utilisateurs sont sollicités, les besoins conscients sont aisément exploitables pour construire l'univers prospectif, parce que les utilisateurs ont - en partie - conscience de ces besoins et peuvent de ce fait les exprimer en amont de la conception. Toutefois, si l'utilisateur peut se représenter certains de ses besoins, il peut aussi ne pas se représenter d'autres besoins potentiels : nous les avons qualifiés de besoins inconscients et de besoins inimaginés. Ces deux derniers sont ainsi plus faciles à construire à partir d'une approche rétrospective, parce que les utilisateurs n'ont pas conscientisé ou exprimé ces besoins en amont de la conception. À partir d'une représentation intermédiaire de l'artefact (par exemple. maquette, prototype), voire d'un autre artefact typique de la technologie ciblée, les utilisateurs peuvent plus aisément les conscientiser et les exprimer. Les utilisateurs découvrent que la technologie offre d'autres moyens d'actions et potentialités que leurs besoins antérieurs (c'est-à-dire conscients a priori). 


\section{CONCLUSION}

L'objectif de cet article était d'éclairer ce qui est sous-jacent à la notion d'utilité dans le contexte de la conception et de l'évaluation ergonomique, particulièrement dans le domaine des technologies émergentes. $\mathrm{Si}$ aujourd'hui beaucoup de travaux portent sur le seul critère de l'utilisabilité, nous suggérons que la notion d'utilité ne peut être négligée dans une démarche ergonomique car, exprimée de façon simpliste, concevoir un produit utilisable ne sert à rien s'il n'est pas utile, avec le risque du rejet pur et simple du produit. Du point de vue de l'ergonomie, il est donc nécessaire de disposer d'une vue complète et de préciser le positionnement de l'utilité par rapport aux autres critères ainsi que des contributions des méthodes couramment mises en œuvre à différentes dimensions de l'utilité.

En ergonomie, l'utilité oscille, comme nous l'avons mis en évidence, entre deux acceptions : l'utilité-destination et l'utilité-valeur. La première acception correspond aux caractéristiques fonctionnelles ou non-fonctionnelles de l'artefact, alors que la seconde correspond aux améliorations ou aux bénéfices significatifs que peut apporter l'artefact aux utilisateurs. Cette "dualité " de l'utilité n'est pas neutre, dans le sens où le lien entre les deux acceptions, bien que souhaité, n'est pas obligatoirement atteint. Elle a nourri notre réflexion, en particulier lorsque nous avons replacé l'utilité au cœur du processus de conception : c'est une propriété construite et évolutive des systèmes qui se caractérise par l'existence d'une dialectique continue entre deux univers, l'un prospectif et l'autre rétrospectif. L'univers prospectif regroupe l'ensemble des particularités, besoins ou solutions pensables (mais pas forcément souhaitables), tandis que l'univers rétrospectif rassemble l'ensemble des expériences pertinentes en termes d'utilité pour informer les concepteurs de l'artefact. De l'amont à l'aval du processus de conception, c'est-à-dire du moment où la forme et l'utilité de l'artefact sont encore indécises jusqu'au moment où la forme finale est éprouvée et continue à évoluer dans l'usage, il est nécessaire d'explorer ces univers. De façon à éviter une exploration trop légère de ceux-ci, il est nécessaire de connaître de façon détaillée aussi bien les méthodes à mettre en œuvre que l'articulation des différents critères de l'ergonomie avec l'utilité.

Dans le processus de conception, la dimension de "besoins " est prédominante. Nous avons montré un lien fort entre cette dimension et celle de l'utilité, même si ces deux dimensions gardent leurs caractéristiques propres. Nous postulons que le besoin constitue l'inspiration d'origine, l'argument et la justification associés à l'utilité d'un artefact au cours du processus de conception. Le caractère plus ou moins conscient des besoins favorise l'un ou l'autre des univers prospectif ou rétrospectif. Si les besoins conscients peuvent être exploités pour construire l'univers prospectif, le recours à des représentations intermédiaires est nécessaire pour faire émerger les besoins latents ou inconscients, ce qui s'enracine davantage dans une approche rétrospective.

Cet article contribue à expliciter la contribution des méthodes à la production d'hypothèses et à l'élaboration d'univers prospectifs, puis à la hiérarchisation, la sélection et la prise de décision, et enfin à l'évaluation de 
l'utilité. Ce dernier apport nous permet de proposer une articulation entre les deux univers et les deux dimensions de l'utilité et le profil des acteurs de la conception. En effet, bien que l'utilité-valeur et l'utilité-destination contribuent à la construction d'univers prospectifs et d'univers rétrospectifs, l'acception de l'utilité, destination ou valeur, détermine leur position privilégiée dans les deux univers. Ainsi, l'utilité-valeur semble plus facile à construire - par les utilisateurs - à partir d'une approche rétrospective, alors que l'utilité-destination est largement exploitable - par des concepteurs - pour construire l'univers prospectif.

Dans ses deux acceptions, l'utilité doit être prise en considération à chaque instant du processus de conception. La mise en relief de la présence des deux univers prospectif et rétrospectif, et l'analyse du rôle des méthodes nous semblent apporter une clarification quant à la prise en compte de l'utilité dans le processus de conception, et quant au positionnement du critère d'utilité par rapport aux autres critères de l'ergonomie. Ce panorama contribue ainsi à enrichir le cadre théorique et méthodologique concernant l'utilité dans la conception des technologies émergentes.

\section{REMERCIEMENTS}

Nous remercions les collectivités territoriales de la Mayenne (CGâ3, Laval Agglomération) qui financent le travail de recherche dans lequel s'inscrit cet article. Nous remercions également Julien Nelson et Olivier Christmann pour leurs remarques constructives qui ont contribué à améliorer l'article. Les auteurs remercient également les experts anonymes de la revue pour l'intérêt porté à ce travail.

\section{RÉRÉRENCES BIBLIOGRAPHIQUES}

Anastassova, M. (2006). L'Analyse ergonomique des besoins en amont de la conception de technologies émergentes: le cas de la Réalité Augmentée pour la formation à la maintenance automobile (thèse de doctorat non publiée). Université Paris Descartes, Paris, France.

Anastassova, M., Burkhardt, J.-M., \& Mégard, C. (2007a). User-centred design and evaluation of augmented reality systems for industrial applications: some deadlocks and breakthroughs. Dans actes de la conférence VRIC'07 Virtual Reality International Conference (pp. 21â-224). Laval, France.

Anastassova, M., Burkhardt, J.-M., Mégard, C., \& Leservot, A. (200â). User-centred design of mixed reality for vehicle maintenance training: an empirical comparison of two techniques for user needs analysis. Dans actes de la conférence HCI International (CDROM), Las Vegas, USA.

Anastassova, M., Burkhardt, J.-M., Megaro, C., \& Ehanno, P. (2007b).L'Ergonomie de la réalité augmentée pour l'apprentissage : une revue. Le Travail humain, $70(2), 97-12 \hat{a}$

Baccino, T., Bellino, C., \& Colombi, T. (200â). Mesure de l'utilisabilité des interfaces. Paris, France : Hermès.

Barcellini, F., Détienne, F., \& Burkhardt, J.-M. (2008). Users and developers mediation in an open source software community: boundary spanning through cross participation in online discussions. International fournal of Human-Computer Studies, 66(7), ââ8-â70. 
Bastien, J. M. C., ï Scapin, D. (2004). La Conception de logiciels interactifs centrée sur l'utilisateur: étapes et méthodes. In P. Falzon (Ed.), Ergonomie (pp. 4囚1-477). Paris, France : Presses universitaires de France.

Beguin, P. (2003). Design as a mutual learning process between users and designers. Interacting with Computers, 15(6), 709-730.

Blandford, A., Connell, I., ï Green, T. R. G. (2004). Concept-based analysis of surface and structural misfits (CASSM) tutorial notes. Récupéré du site de l'auteur http://www.uclic.ucl.ac.uk/annb/CASSM/downloadables/CASSMtutorial.pdf

Blandford, A., Green, T. R. G., Furniss, D., i Makri, S. (2007). Evaluating system utility and conceptual fit using CASSM. International fournal of HumanComputer Studies, 66(6), 1-26.

Boehm, B.W. (1986). A Spiral Model of Software Development and Enhancement. SIGSOFT Software Engineering Notes, 11(4), 14-24.

Bonnardel, N., i Marmèche, E. (200囚). Towards supporting evocation processes in creative design: A cognitive approach. International fournal of Human-Computer Studies, 63(4-区), 422-43区.

Bourmaud, G. (2007). L'organisation systémique des instruments : méthodes d'analyse, propriétés et perspectives de conception ouvertes. Communication présenteé au Colloque de l'Association pour la Recherche Cognitive - ARCo'07. Nancy, France. Récupéré des archives ouvertes de l'INRIA : http://hal.archives-ouvertes. fr/inria-00191128/

Brangier, E., ï Bastien, J. M. C. (2006). L'analyse de l'activité est-elle suffisante et/ou pertinente pour innover dans le domaine des nouvelles technologies? In G. Valléry $\ddot{i}$ R. Amalberti (Eds.), L'Analyse du travail en perspectives. Influences et évolutions (pp. 143-1囚6). Toulouse, France : Octarès.

Brangier, É., Hammes-Adelé, S., ï Bastien, J.-M. C. (2010). Analyse critique des approches de l'acceptation des technologies: de l'utilisabilité à la symbiose humain-technologie-organisation. Revue Européenne de Psychologie Appliquée, 60(2), 129-146.

Burkhardt, J.-M. (1997). Réutilisation de solutions en conception orientée-objet : un modèle cognitif des mécanismes et représentations mentales (thèse de doctorat non publiée). Université Paris Descartes, Paris, France.

Burkhardt, J.-M. (2006). L'homme et son environnement: Ergonomie, Facteurs-Humains et Réalité Virtuelle. In P. Fuchs, G. Moreaux, A. Berthoz ï J.-L.Vercher (Eds.), Le Traité de la RéalitéVirtuelle (Vol. 1, pp. 117-1â0). Paris, France : Presses de l'École des Mines de Paris.

Burkhardt, J.-M., ï Sperandio, J.-C. (2004). Ergonomie et conception informatique. In P. Falzon (Ed.), Ergonomie (pp. 437-4â0). Paris, France : Presses universitaires de France.

Coughlan, J., ï Macredie, R. (2002). Effective Communication in Requirements Elicitation: A Comparison of Methodologies. Requirements Engineering, 7(2), 47-60.

Cockton, G., Woolrych, A., ï Lavery, D. (2008). Inspection-based evaluations. In J. A. Jacko $\ddot{i}$ A. Sears (Eds.). The Human-Computer Interaction Handbook. Fundamentals, Evolving Technologies, and Emerging Applications (Second ed., pp. 1171-1190). New York, USA : Lawrence Erlbaum Associates.

Couix, S. (2007). Usages et construction des modèles de tâches dans la pratique de l'ergonomie : une étude exploratoire (thèse de Master non publiée). Université Paris Descartes, Paris, France.

Daniellou, F. (1992). Le Statut de la pratique et des connaissances dans l'intervention ergonomique de conception (thèse d'Habilitation à Diriger des Recherches non publiée). Université Le Mirail, Toulouse, France.

Darses, F., i Wolff, M. (2006). How do designers represent to themselves the users' needs? Applied Ergonomics, 37(6), 7â7-764. 
Davis, F. D. (1989). Perceived usefulness, perceived ease of use, and user acceptance of information technology. MIS Quarterly, 13(3), 319-340.

Diez, E., $\ddot{1}$ McIntosh, B. S. (2009). A review of the factors which influence the use and usefulness of information systems. Environmental Modelling E Software, 24(â), â 88-602.

Gaudart, C. (2000). Quand l'écran masque l'expérience des opérateurs vieillissants changement de logiciel et activité de travail dans un organisme de services. PISTES, 3(2), 1-16. Récupéré sur le site de la revue : http://www.pistes.uqam. $\mathrm{ca} / \mathrm{v} 2 \mathrm{n} 2 / \mathrm{pdf} / \mathrm{v} 2 \mathrm{n} 2 \mathrm{a} 4 . \mathrm{pdf}$

Grudin, J. (1992). Utility and usability: research issues and development contexts. Interacting with Computers, 4(2), 209-217.

Haradji, Y., ï Faveaux, L. (2006). Évolution de notre pratique de conception (198â-200â) : modéliser pour mieux coopérer à partir des critères d'utilité, d'utilisabilité. @ctivités - Revue électronique, 3(1), 67-98. Récupéré à partir du site de revue : www.activites.org/v3n1/haradji.pdf

Hornbæk, K. (200â). Current practice in measuring usability: challenges to usability studies and research. International fournal of Human-Computer Studies, 64(2), 79-102.

Johnson, H., ï Carruthers, L. (2006). Supporting creative and reflective processes. International fournal Human-Computer Studies, 64(10), 998-1030.

Jolly, C., Florin, A., Nocus, I., Delalande, J., Bréé, J., Saltet, J., et al. (2008). Comment vivront les enfants de demain? Cholet, France : Le Pôle Enfant.

Kaiya, H., Shinbara, D., Kawano, J., ï Saeki, M. (200â). Improving the detection of requirements discordances among stakeholders. Requirements Engineering, 10(4), 289-303.

Keil, M., Beranek, P. M., ï Konsynski, B. R. (199â). Usefulness and ease of use: field study evidence regarding task considerations. Decision Support Systems, 13(1), 7â-91.

Lindgaard, G., ï Parush, A. (2008). Utility and experience in the evolution of usability. In E. Law, E. Hvannberg ï G. cockton (Eds.), Maturing Usability. Quality in Software, Interaction andValue (pp. 222-240). London, UK : SpringerVerlag.

Loup-Escande, E., Burkhardt, J.-M., Christofol, H., ï Richir, S. (2010). 3D Child Software: a decision-making help tool in innovative product design. Fournal of Decision Systems, 19(1), 9-31.

Maguire, M. (2001). Methods to support human-centred design. International Fournal of Human-Computer Studies, 55(4), â87-634.

Mich, L., Anesi, C., $\ddot{i}$ Berry, D. M. (2004). Requirements Engineering and Creativity: An Innovative Approach Based on a Model of the Pragmatics of Communication. Communication à la conférence Requirements Engineering: Foundation of Software Quality (REFSQ'04), Riga, Lettonie. Récupéré sur le site de l'Université de Waterloo : http://se.uwaterloo.ca/ dberry/FTP_SITE/reprints. journals.conferences/MichAnesiBerry2004Creativity.pdf

Mollo, V., ï Falzon, P. (2004). Auto- and allo-confrontation as tools for reflective activities. Applied Ergonomics, 35(6), â31-â40.

Neumann, J., ï Morgenstern, O. (1947). Theory of Games and Economic Behavior. Princeton, NJ, USA : Princeton University Press.

Newell, A. F., Carmichael, A., Morgan, M., ï Dickinson, A. (2006). The use of theatre in requirements gathering and usability studies. Interacting with Computers, 18(â), 996-1011.

Nguyen, L., ï Shanks, G. (2009). A framework for understanding creativity in requirements engineering. Information and Software Technology, 51(3), 6ââ-662.

Nicolas, L. (2000). La "Simulation Langagière " en Analyse Fonctionnelle : entre travail des concepteurs et travail des ergonomes. Communication au XXXV Congrès de la SELF, Toulouse, France. 
Nielsen, J. (1993). Usability Engineering. San Francisco, CA, USA : Morgan Kaufmann. Nielsen, J., ̈̈ Mack, R. L. (Eds.). (1994). Usability Inspection Methods. New York, NY, USA : John Wiley $\ddot{i}$ Sons.

Olivier, R. (1980). A cognitive model of the antecedents and consequences of satisfaction decisions. Fournal of Marketing Research, 17(3), 460-469.

Plos, O., Buisine, S., Aoussat, A., ï Dumas, C. (2007). Analysis and translation of user needs for assistive technology design. Communication à International Conference on Engineering Design (ICED’07), Paris, France. Récupéré du site du second auteur : http://stephanie.buisine.free.fr/publis/ICED07.pdf

Premkumar, G., ï Bhattacherjeeb, A. (2006). Explaining information technology usage: a test of competing models. Omega - The International fournal of Management Science, 36(1), 64-7区.

Prospective (n.d). Dans Trésor de la Langue Française. Récupéré de http://atilf. atilf.fr

Rabardel, P., ï Beguin, P. (200â). Instrument mediated activity: from subject development to anthropocentric design. Theoretical Issues in Ergonomic Science, 6(â), 429-461.

Richir, S. (2003). Les technologies de la réalité virtuelle pour la conception de produits (thèse d'Habilitation à Diriger des Recherches non publiée). Université d'Angers, Angers, France.

Robertson, S. (2001). Requirements trawling: techniques for discovering requirements. International fournal of Human-Computer Studies, 55(4), 40â-421.

Rothwell, R. (1994). Towards the fifth-generation innovation process. International Marketing Review, 11(1), 7-31.

Scapin, D., i Bastien, J.-M. C. (1997). Ergonomic criteria for evaluating the ergonomic quality of interactive systems. Behaviour and Information Technology, 16(4-â), pp. 220-231

Scholtz, J., Morse, E., i Steves, M.P. (2006). Evaluation metrics and methodologies for user-centered evaluation of intelligent systems. Interacting with Computers, 18(6), 1186-1214.

Sheth, J. (1973). A model of industrial buyer behavior. Fournal of Marketing, 37, â0-â 6 .

Silius, K., ï Tervakari, A.-M. (2003). The usefulness of web-based learning environments: the evaluation tool into the portal of finnish virtual university. Communication présentée à International Conference on Network Universities and e-learning, Valencia, Spain. Récupéré sur le site de l'Université de Jyväskylä : http://www.mit.jyu.fi/OPE/kurssit/TIES462/Materiaalit/Silius_Tervakari.pdf

Sperandio, J.-C. (2001). Critères ergonomiques de l'assistance technologique aux opérateurs. Communication au congrès JIM'2001 : Interaction Homme - Machine i Assistance, Metz, France.

Spreng, R., Mackenzie, S., ï Olshavsky, R. (1996). A reexamination of the determinants of consumer satisfaction. Fournal of marketing, 60(6), 1â-32.

Sternberg, R. J. (200â). Creativity or creativities? International fournal HumanComputer Studies, 63(4-â), 370-382

Tohidi, M., Buxton, W., Baecker, R., ï Sellen, A. (2006). Getting the Right Design and the Design Right: Testing Many Is Better Than One. In actes de la conference CHI 2006 - ACM Conference on Human Factors in Computing (pp. 1243-12â2). Quebec, Montreal Canada : ACM press. Récupéré du site du deuxième auteur : www.billbuxton.com/rightDesign.pdf

Tricot, A., Plégat - Soutjis, F., Camps, J.-F., Amiel, A., Lutz G., ï Morcillo, A. (2003). Utilité, utilisabilité, acceptabilité : interpréter les relations entre trois dimensions de l'évaluation des EIAH. Communication à la conférence EIAH 2003, Strasbourg, France. 

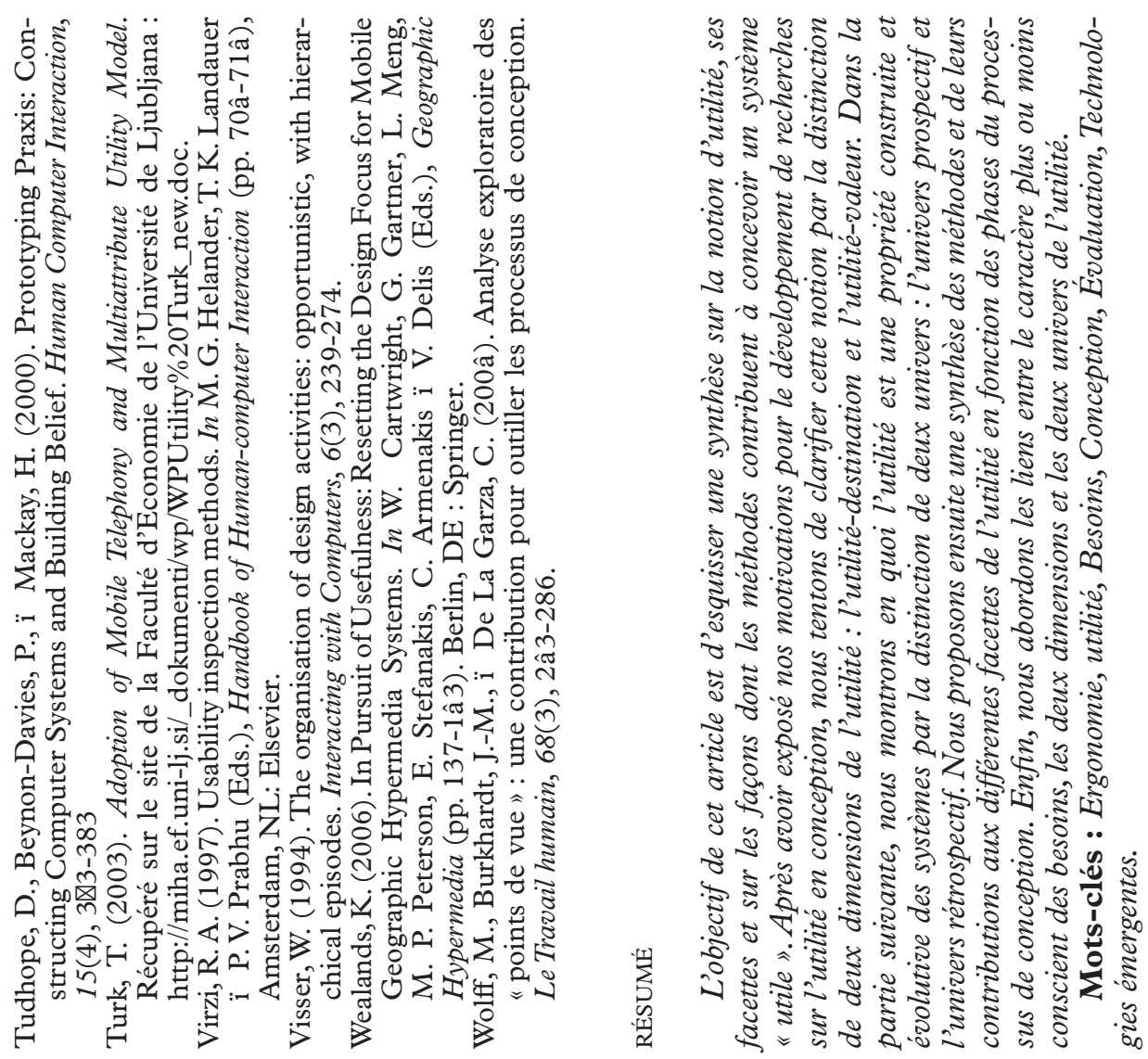